\title{
Interleukin-6 trans-signaling increases the expression of carcinoembryonic antigen- related cell adhesion molecules 5 and 6 in colorectal cancer cells
}

\author{
Reinhild Holmer ${ }^{1}$, Georg H. Wätzig ${ }^{2}$, Sanjay Tiwari ${ }^{3}$, Stefan Rose-John ${ }^{4}$ and Holger Kalthoff ${ }^{*}$
}

\begin{abstract}
Background: Colorectal cancer (CRC) is among the five most frequent causes for cancer-related deaths in Europe. One of the most important tumor-associated antigens for CRC is carcinoembryonic antigen-related cell adhesion molecule 5 (CEACAM5), which is involved in cell adhesion, migration, anoikis, tumor invasion and metastasis. Its family member CEACAM6 is also upregulated in adenomas and carcinomas of the colon and an independent predictor of poor survival. Previous studies have reported a link between upregulation of CEACAM5 and interleukin-6 (IL-6). IL-6 plays an important role in CRC progression, and signaling is mediated via two pathways (classic and trans-signaling). However, this link could not be confirmed by other studies, and the role of IL-6 trans-signaling in the CEACAM5 upregulation has not been elucidated. Moreover, the impact of IL-6 on the expression of CEACAM6 has not yet been examined.
\end{abstract}

Methods: The expression of IL-6, IL-6 receptor (IL-6R), glycoprotein (gp) 130, CEACAM5 and CEACAM6 was analyzed by RT-PCR, Western blot, flow cytometry or qPCR. Colon cell lines were incubated with IL-6 or Hyper-IL-6 (mediating IL-6 trans-signaling), and subsequently, the expression of CEACAMs was determined by qPCR or Western blot. FLLL31, an inhibitor of the phosphorylation of signal transducer and activator of transcription-3 (STAT3), was used to determine the role of STAT3 phosphorylation.

Results: We confirmed that colon carcinoma cell lines express IL-6 and IL-6R. We observed only a weak upregulation of CEACAM5 and CEACAM6 by classic IL- 6 signaling, but a strong increase by IL-6 trans-signaling. This upregulation depended on the phosphorylation of STAT3.

Conclusions: Our data show the upregulation of the tumor-associated antigens CEACAM5/6 by trans-signaling of the pro-inflammatory cytokine IL-6. This mechanism may contribute to the tumor-promoting role of IL-6 and could therefore be a target for therapeutic intervention in particular by specific inhibitors such as sgp130Fc.

Keywords: IL-6, Hyper-IL-6, Trans-signaling, CEA, Inflammation, Tumor-associated antigens, Tumor marker, Colon cancer, Colitis-associated cancer

\footnotetext{
*Correspondence: hkalthoff@email.uni-kiel.de

'Division of Molecular Oncology, Institute for Experimental Cancer Research, University Hospital Schleswig-Holstein, 24105 Kiel, Germany

Full list of author information is available at the end of the article
} 


\section{Background}

CRC is still one of the leading causes of cancer deaths in Europe. According to calculations for the year 2014, it ranks second in men and third in women [1]. Several risk factors exist, including smoking, alcohol consumption, diabetes and inflammation [2,3]. The link between inflammation and tumorigenesis is exemplified by patients with colitis-associated cancer (CAC). These are CRC patients that have previously suffered from inflammatory bowel disease (IBD). It is well-known that IBD patients have a higher risk of developing CAC/CRC $[4,5]$.

One of the key cytokines in IBD as well as in CRC is IL-6 [6]. IL-6 is a pleiotropic cytokine involved in various processes of innate and adaptive immunity $[7,8]$. In the classic IL-6 signaling pathway, IL-6 binds to the membrane-bound IL-6R, which subsequently transmits the signal via the recruitment and homodimerization of two gp130 subunits. Consequently, an intracellular cascade is activated involving STAT3, mitogen-activated protein kinase (MAPK) and phosphatidylinositol-4,5bisphosphate 3-kinase (PI3K) activation [9]. Whereas gp130 is ubiquitously expressed, IL-6R expression is restricted to only a few cell types, such as hepatocytes and certain leukocytes. However, a soluble form of IL-6R (sIL$6 \mathrm{R}$ ) is generated by protease-mediated receptor shedding from the membrane or by alternative splicing. In contrast to some other soluble receptors, the sIL-6R does not act as an antagonist. Instead, it binds to IL-6 and transactivates cells that only express gp130. This process was termed trans-signaling [9]. It is selectively inhibited by a naturally occuring soluble form of gp130 (sgp130). This knowledge was used to generate a potent and selective inhibitor of trans-signaling by fusing the sgp 130 protein to the Fc part of a human IgG1 antibody. The resulting fusion protein is called sgp130Fc [9] and the optimized variant FE 999301 has already entered clinical development for the treatment of inflammatory bowel disease.

Several studies demonstrated a significant role of IL-6 in IBD as well as in CRC. These studies were recently reviewed by Waldner and Neurath, who concluded that IL-6 is the "master regulator of intestinal disease" [6]. Interestingly, in most studies, the pro-inflammatory and tumor-promoting activity of IL-6 was mediated via IL-6 trans-signaling $[6,10]$.

A causal link between IL-6 and CEACAM5 is revealed by significant association of serum levels of IL-6 with high serum levels of CEACAM5 [11, 12]. CEACAM5 (also called carcinoembryonic antigen, CEA) is one of the best-known tumor-associated antigens for CRC [13-15]. It is expressed in normal mucosal cells of the colon, but overexpressed in adenocarcinomas of the colon. In addition, its serum levels are elevated in CRC patients [15]. CEACAM5 is an adhesion molecule that was shown to be involved in cell adhesion, migration, anoikis, tumor invasion and metastasis [16, 17]. Furthermore, it activates inhibitory CEACAM1 signaling in natural killer cells (NK cells) and thereby blocks the cytotoxicity of NK cells [17]. CEACAM6, another family member of the carcinoembryonic antigen family, is already upregulated in benign precursor lesions like hyperplastic colorectal polyps and early adenomas [18]. Moreover, CEACAM6 is an independent predictor of poor survival for CRC patients [19] and is involved in tissue architecture and colonocyte differentiation [20].

IL-6 was previously shown to increase the expression of CEACAM5 on some CRC cells [21, 22]. However, this relationship was not observed for all CRC cell lines, and another study only found a very small and not significant stimulatory effect of IL-6 on the CEACAM5 expression [23]. To our knowledge, no study has yet examined the relationship between IL-6 trans-signaling and CEACAM5 and CEACAM6. Thus, the aim of this study was to systematically analyze the impact of IL-6 classic and trans-signaling on the expression of CEACAM5 and CEACAM6 in colorectal cancer cells.

\section{Methods}

\section{Cell culture and proteins}

The human colorectal adenocarcinoma cell lines HT29 (called HT29p for 'parental' cells to distinguish it from other HT29 derivatives in our laboratory) and SW480 were obtained from the American Type Culture Collection (ATCC). HT29c cells had been generated in our laboratory by repeated injection of HT29 cells into the portal venous system of nude rats, subsequent isolation from liver metastases and reculturing in vitro $[24,25]$. Colo357 cells, derived from a metastasis of a pancreatic adenocarcinoma, were a kind gift of Dr. R. Morgan (Denver, CO) [26]. These cells were routinely cultured in Roswell Park Memorial Institute (RPMI)-1640 medium (Gibco/Life Technologies, Darmstadt, Germany) supplemented with $10 \%$ fetal bovine serum (FBS, PAN-Biotech, Aidenbach, Germany), $1 \mathrm{mM}$ sodium pyruvate (Gibco) and $2 \mathrm{mM}$ glutaMAX (Gibco). The human colorectal adenocarcinoma cell line Caco-2 was obtained from ATCC. It was cultured in Dulbecco's Modified Eagle's Medium (DMEM) (Gibco) supplemented with $10 \%$ FBS, $1 \mathrm{mM}$ sodium pyruvate and $2 \mathrm{mM}$ glutaMAX. The normal mucosa-derived colon cell lines CSC1 [27] and NCM460 [28] were a kind gift of Dr. Mary Pat Moyer (San Antonio, TX, USA). These cell lines were maintained in M3 Base cell culture medium complete (M300A-500, Incell, San Antonio, TX, USA) with $10 \%$ FBS. Ba/F3-gp130/IL-6R cells are Ba/F3 pre-B cells lacking endogenous gp130, which had been stably transfected with IL-6R and gp130 as a model system for IL-6 signaling $[29,30]$. They were cultured in DMEM high glucose medium (Gibco) supplemented with $10 \%$ FBS, $1 \mathrm{mM}$ sodium pyruvate, $2 \mathrm{mM}$ glutaMAX and $1 \mathrm{ng} / \mathrm{ml}$ IL-6. 
IL-6 and Hyper-IL-6 - a fusion protein of IL-6 and sIL-6R mimicking the IL-6 trans-signaling complex were produced by the group of Prof. Stefan RoseJohn as previously described [31, 32]. All cells were maintained at $37{ }^{\circ} \mathrm{C}$ in a humid atmosphere with $5 \%$ $\mathrm{CO} 2$ and routinely checked for mycoplasma contamination with the MycoTrace kit (PAA/GE Healthcare, Cölbe, Germany).

\section{RNA isolation and CDNA synthesis}

RNA was isolated using the RNeasy Plus Mini Kit (Qiagen, Hilden, Germany). RNA concentration was measured in a Nanodrop spectrophotometer (Thermo Fisher Scientific, Dreieich, Germany) and quality-checked on a $1 \%$ agarose gel. $2 \mu \mathrm{g}$ of RNA were reverse-transcribed into cDNA using the Maxima First Strand cDNA Synthesis Kit (Thermo Fisher Scientific).

Reverse transcriptase polymerase chain reaction (RT-PCR) PCR was performed using the Dream Taq Green Polymerase (Thermo Fisher Scientific). The primer sequences are depicted in Table 1, and the following conditions were used: initial denaturation: $95^{\circ} \mathrm{C}, 2 \mathrm{~min}$; denaturation: $95^{\circ} \mathrm{C}, 30 \mathrm{~s}$; annealing: $60{ }^{\circ} \mathrm{C}, 30 \mathrm{~s}$; extension: $72{ }^{\circ} \mathrm{C}, 1 \mathrm{~min}$ (40 cycles); final extension: $72{ }^{\circ} \mathrm{C}, 10 \mathrm{~min}$. The PCR product was analyzed by agarose gel electrophoresis on a $2 \%$ agarose gel.

\section{Quantitative real-time polymerase chain reaction (qPCR)}

cDNA was diluted 100-fold in nuclease-free water. $2 \mu \mathrm{l}$ of diluted cDNA were used in a $20 \mu \mathrm{l}$ reaction with FastSybr Green mastermix (Applied Biosystems/Life Technologies). The primer sequences are depicted in Table 2, and the following conditions were used: initial denaturation: $95{ }^{\circ} \mathrm{C}, 20 \mathrm{~s}$; denaturation: $95{ }^{\circ} \mathrm{C}, 3 \mathrm{~s}$; annealing/extension: $60{ }^{\circ} \mathrm{C}, 30 \mathrm{~s}$ (usually 40 cycles). Specificity of the product was verified by melt curve analysis and agarose gel electrophoresis.
Phorbol-12-myristate-13-acetate (PMA) stimulation and enzyme-linked immunosorbent assays (ELISAs)

For PMA stimulation, HT29p cells were seeded in a 96-well plate. On the next day, the medium was changed to remove non-adherent or dead cells. After $72 \mathrm{~h}$, the supernatants were collected to measure the baseline (unstimulated) sIL-6R production of the cells (data not shown). Subsequently, the medium was changed, and the cells were stimulated for $2 \mathrm{~h}$ at room temperature (RT) with medium containing either $100 \mathrm{nM}$ PMA (Calbiochem/Merck, Darmstadt, Germany) dissolved in dimethyl sulfoxide (DMSO) or $0.5 \%$ DMSO as solvent control in triplicate wells. Supernatants from the triplicate wells were harvested and centrifuged for $15 \mathrm{~min}$ at $16,000 \mathrm{xg}$ and $4{ }^{\circ} \mathrm{C}$ to remove cells and cellular debris. The purified supernatants were stored at $-80{ }^{\circ} \mathrm{C}$ until ELISA analysis. sIL-6R concentrations were measured using ELISA kits (R\&D Duoset, R\&D Systems, Wiesbaden, Germany) according to the manufacturer's instructions.

\section{Analysis of STAT3 phosphorylation and CEACAM expression by Western blotting}

To analyze the phosphorylation of STAT3 and CEACAM5/6, HT29p cells were seeded in 6-well plates. After $48 \mathrm{~h}$, the medium was replaced by serum-free medium. The next morning, cells were stimulated with different concentrations of IL-6 or Hyper-IL-6, a fusion protein of IL-6 and sIL-6R mimicking the IL-6 trans-signaling complex (see above). After $15 \mathrm{~min}$ (STAT3) or $48 \mathrm{~h}$ (CEACAM5/6), the cells were lysed with radioimmunoprecipitation assay (RIPA) buffer and stored at $-20{ }^{\circ} \mathrm{C}$ until analysis for STAT3 phosphorylation in Western blots.

For Western blots, the lysates were thawed on ice, sonicated and centrifuged $\left(13,000 \mathrm{rpm}, 15 \mathrm{~min}, 4{ }^{\circ} \mathrm{C}\right)$ to remove cellular debris. Protein concentration was determined with the DC assay (Bio-Rad Laboratories, Munich, Germany). Equal amounts of protein were loaded onto a 4-20 \% tris-glycine gel (Life Technologies) and separated by SDS-PAGE. Proteins were blotted on a PVDF membrane (Immobilon-FL; Millipore/Merck,

Table 1 Primers used for RT-PCR

\begin{tabular}{llll}
\hline Name & Sequence & Amplicon [bp] (mRNA/genomic) & Reference \\
\hline hu_mIL6R_For & CATTGCCATTGTTCTGAGGTTC & 280/not amplified & [54] \\
hu_IL6R_Rev & GTGCCACCCAGCCAGCTATC & 278 (mll6R), 280 (sll6R)/not amplified & [54] \\
hu_gp130C & GGTACGAATGGCAGCATACA & $713 / 10170$ & [55] \\
hu_gp130D & CTGGACTGGATTCATGCTGA & $713 / 10170$ & [55] \\
hu_IL6_For1 & TCCACAAGCGCCTTCGGTCC & $621 /$ not amplified & primerblast \\
hu_IL6_Rev1 & TTGCCGAAGAGCCCTCAGGCT & $621 /$ not amplified & primerblast \\
hu_RPL22_For & TCGCTCACCTCCCTTTCTAA & $250 / 6652$ & [56] \\
hu_RPL22_Rev & TCACGGTGATCTTGCTCTTG & 250/6652 & [56] \\
\hline
\end{tabular}

$R P L 22$ ribosomal protein L22 
Table 2 Primers used for $\mathrm{qPCR}$

\begin{tabular}{llll}
\hline Name & Sequence 5'-3' & Amplicon [bp] (mRNA/genomic) & Reference \\
\hline hu_CEA_For & CTTATCGCCAAAATCACGC & $138 / 6195$ & http://primerdepot.nci.nih.gov \\
hu_CEA_Rev & CCAGCTGAGAGACCAGGAGA & $138 / 6195$ & http://primerdepot.nci.nih.gov \\
hu_CEACAM6_For1 & GCATGTCCCCTGGAAGGA & $179 / 1076$ & {$[57]$} \\
hu_CEACAM6_Rev1 & CGCCTTTGTACCAGCTGTAA & $179 / 1076$ & {$[57]$} \\
hu_RPL22_For & TCGCTCACCTCCCTTTCTAA & $250 / 6652$ & {$[56]$} \\
hu_RPL22_Rev & TCACGGTGATCTTGCTCTTG & $250 / 6652$ & {$[56]$} \\
hu_PPIC_For & GGAAAAGTCATTGATGGGATG & $127 / 1907$ & sequence from Eva Simon, Kiel \\
hu_PPIC_Rev & CAAAAGGCGTTTCACGTCTA & $127 / 1907$ & sequence from Eva Simon, Kiel \\
hu_SDHA_For & ATTTGGTGGACAGAGCCTCA & $126 /$ not amplified & sequence from Eva Simon, Kiel \\
hu_SDHA_Rev & CTGGTATCATATCGCAGAGACCT & $126 /$ not amplified & sequence from Eva Simon, Kiel \\
\hline
\end{tabular}

RPL22 ribosomal protein L22, PPIC peptidylprolyl isomerase C, SDHA succinate dehydrogenase complex, subunit A, flavoprotein

Darmstadt, Germany), blocked with $5 \%$ milk or bovine serum albumin for $1 \mathrm{~h}$ at RT and incubated with the primary antibody overnight at $4{ }^{\circ} \mathrm{C}$. Secondary antibody incubation was performed for $1 \mathrm{~h}$ at RT. All washes were performed with TBS supplemented with $0.01 \%$ Tween- 20 . Blots were dried with methanol and scanned in an Odyssey imager (LI-COR, Bad Homburg, Germany). Alternatively, horseradish peroxidase (HRP)-coupled secondary antibodies were used. After incubation, these membranes were incubated with a substrate for electrochemiluminescence (ECL), and readout was performed using films (Amersham Hyperfilm ECL, both from GE Healthcare, Munich, Germany) and an Agfa Curix 60 developing machine (Agfa, Mortsel, Belgium). The following antibodies were used: P-STAT3 (\#9131, Cell Signaling Technology/New England Biolabs, Schwalbach, Germany), STAT3 (\#9139, Cell Signaling Technology), $\beta$-actin (ab6276, Abcam, Cambridge, UK), goat-antimouse-IRDye680 (LI-COR), goat-anti-rabbit-IRDye800CW (LI-COR), CEACAM5 (T84.66, kindly provided by Stefanie Nittka, Mannheim, Germany), CEACAM6 (AM02001PU$\mathrm{N}$, Acris, Herford, Germany), goat-anti-rabbit IgG-HRP (\#7074, Cell Signaling) and horse-anti-mouse IgG-HRP (\#7076, Cell Signaling).

\section{CEACAM analysis by flow cytometry}

For the analysis of CEACAM molecules by flow cytometry, cells were harvested after Accutase treatment (GE Healthcare, Munich, Germany). Subsequently, all steps were performed on ice. Cells were washed with FACS buffer (PBS with $2 \%$ human serum, 2 mM EDTA and $0.02 \%$ sodium azide), blocked with FACS buffer for $15 \mathrm{~min}$ and stained with primary antibodies diluted in FACS buffer (30 min on ice). Subsequently, they were washed three times with FACS buffer and incubated with the fluorochrome-coupled secondary antibody. After washing, the cells were incubated with FACS buffer containing 7AAD (BD Bioscience, Franklin Lakes, NJ, USA).
Afterwards, the cells were measured in a FACScalibur (BD). Weasel software v3.0 (chromocyte, Sheffield, UK) was used for data analysis. Dead cells were excluded by gating for the 7AAD-negative cells, as dead cells were previously shown to be false positive for CEACAM5/6. The following antibodies were used: CEACAM5 (C1P83, produced by our group as previously described [33, 34]), CEACAM6 (AM02001PU-N, Acris, Herford, Germany), mouse IgG1-isotype control (X0931, Dako, Glostrup, Denmark) and anti-mouse-IgG-Alexa488 (Invitrogen/Life Technologies, Darmstadt, Germany).

\section{Ethics statement}

Our study is in compliance with the Helsinki Declaration. We did not perform a clinical trial or used any materials from clinical specimens and therefore, no consent of patients was necessary. Only the cell lines used in this work were obtained from the sources indicated in the "Cell culture and protein" section.

\section{Results}

Colon cell lines express molecules mediating IL-6 signaling

To understand the impact of IL- 6 on colon cells, we first analyzed by reverse transcription polymerase chain reaction (RT-PCR) whether mucosa-derived colon cell lines (CSC1, NCM460) and colorectal cancer cell lines (Caco-2, HT29p, HT29c, SW480) express key components of the IL-6 signaling pathway. The mucosa-derived cell lines were originally isolated from histologically normal colonic margins from patients undergoing resection for colon adenocarcinomas and represent the disease in its early stages of transformation [27, 35]. Most cell lines expressed IL-6 mRNA. However, the level among the cell lines varied, and the parental HT29 cell line (HT29p) did not show any IL-6 expression (Fig. 1a). In contrast, the cell line HT29c, which was derived from HT29p cells using a successive intrahepatic selection procedure in rats [24, 25], expresses IL-6 (Fig. 1a). Moreover, all cell 

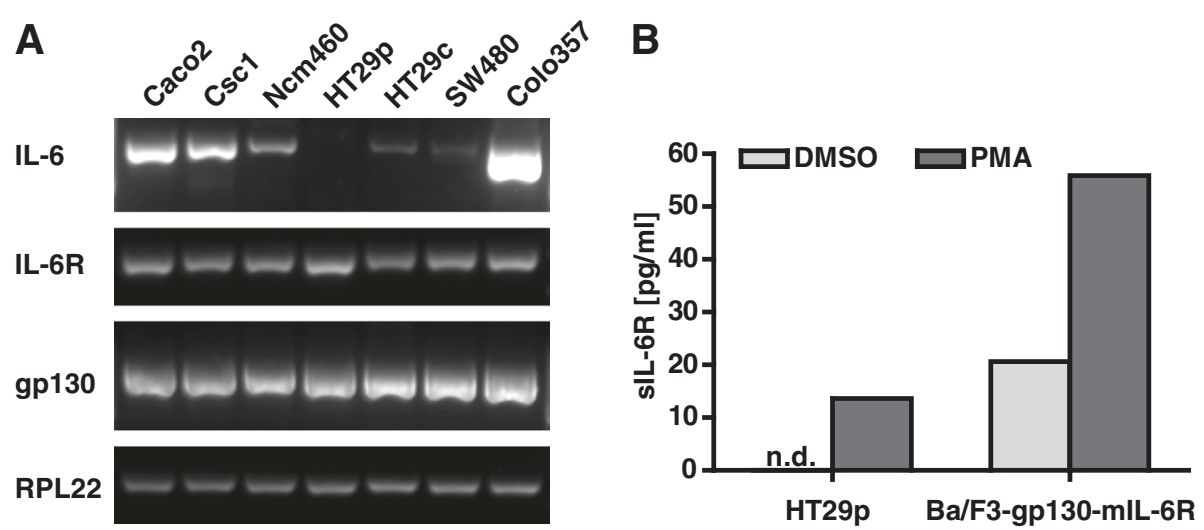

Fig. 1 Colon cells express IL-6, IL-6R and gp130. a, RNA from different colorectal cancer cells (Caco-2, HT29p, HT29c, SW480) and normal mucosa-derived colon cells (CSC1, NCM460) was extracted, reverse-transcribed into CDNA and analyzed for the expression of IL-6, IL-6R and gp130. The pancreatic cell line Colo357 served as a positive control. Ribosomal protein L22 (RPL22) was used as a reference gene to monitor equal transcription of cDNA. b, HT29p cells were seeded in 6-well plates. After $72 \mathrm{~h}$, the medium was replaced by medium containing either the solvent dimethyl sulfoxide (DMSO) or phorbol-12-myristate-13-acetate (PMA) in DMSO. Supernatants were collected after $2 \mathrm{~h}$, and the sll-6R concentration was determined by ELISA. Ba/F3-gp130-mlL-6R cells were used as a positive control. n.d. not detected

lines showed mRNA expression of the IL-6R and of the co-receptor gp130.

However, we did not detect the IL-6R on the surface of colorectal cancer cells by flow cytometry (data not shown). Therefore, we assumed that either the expression level was low or that the receptor was shed. IL-6R is mainly shed by ADAM17, which is strongly activated by phorbol-12-myristate-13-acetate (PMA) [36]. Stimulation of HT29p cells with PMA led to an increase of the sIL-6R concentration in the supernatant (Fig. 1b), demonstrating that colorectal cancer cells express the IL-6R protein on their membrane, and that it can be shed from the surface.

\section{CEACAM5 and CEACAM6 are expressed in most colon} cell lines

Before we analyzed the relationship between IL-6 and CEACAMs, we examined the expression of CEACAM5 and CEACAM6 in the normal mucosa-derived and colorectal cancer cell lines. Most of these cell lines expressed CEACAM5 as well as CEACAM6. However, the level varied between different cell lines. On the mRNA level, HT29p and NCM460 cells showed the highest expression of CEACAM5 (Fig. 2). HT29p also had the highest mRNA level of CEACAM6. Interestingly, the variant HT29c showed a much lower expression of CEACAM5 and CEACAM6 than HT29p. The cell line Caco-2
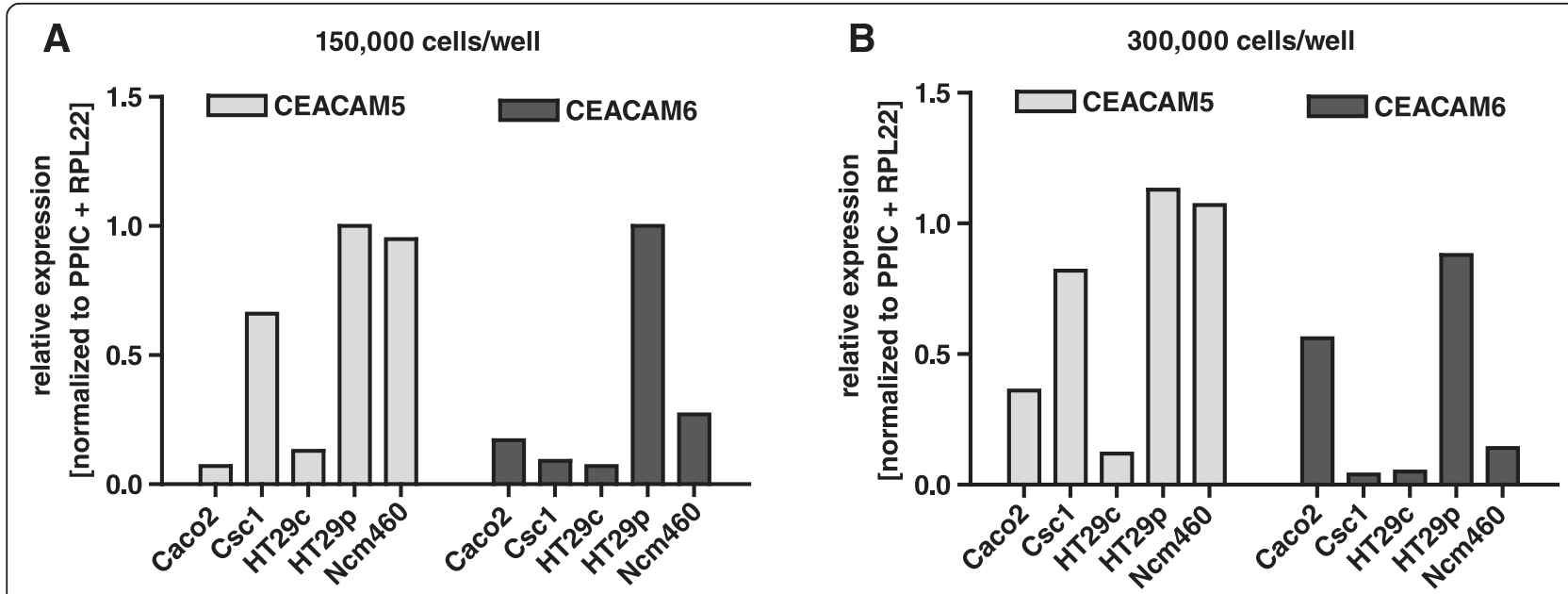

Fig. 2 Most colon cell lines express mRNA of CEACAM5 and CEACAM6. a, 150,000 or 300,000 (b) colorectal cancer cells (Caco-2, HT29p, HT29c, SW480) or normal mucosa-derived colon cell lines (CSC1, NCM460) were seeded in 6-well plates. After 48 h, the RNA was extracted and analyzed by qPCR for the expression of CEACAM5 and CEACAM6. PPIC and RPL22 were used as reference genes. SW480 cells did not show any expression (not shown) 


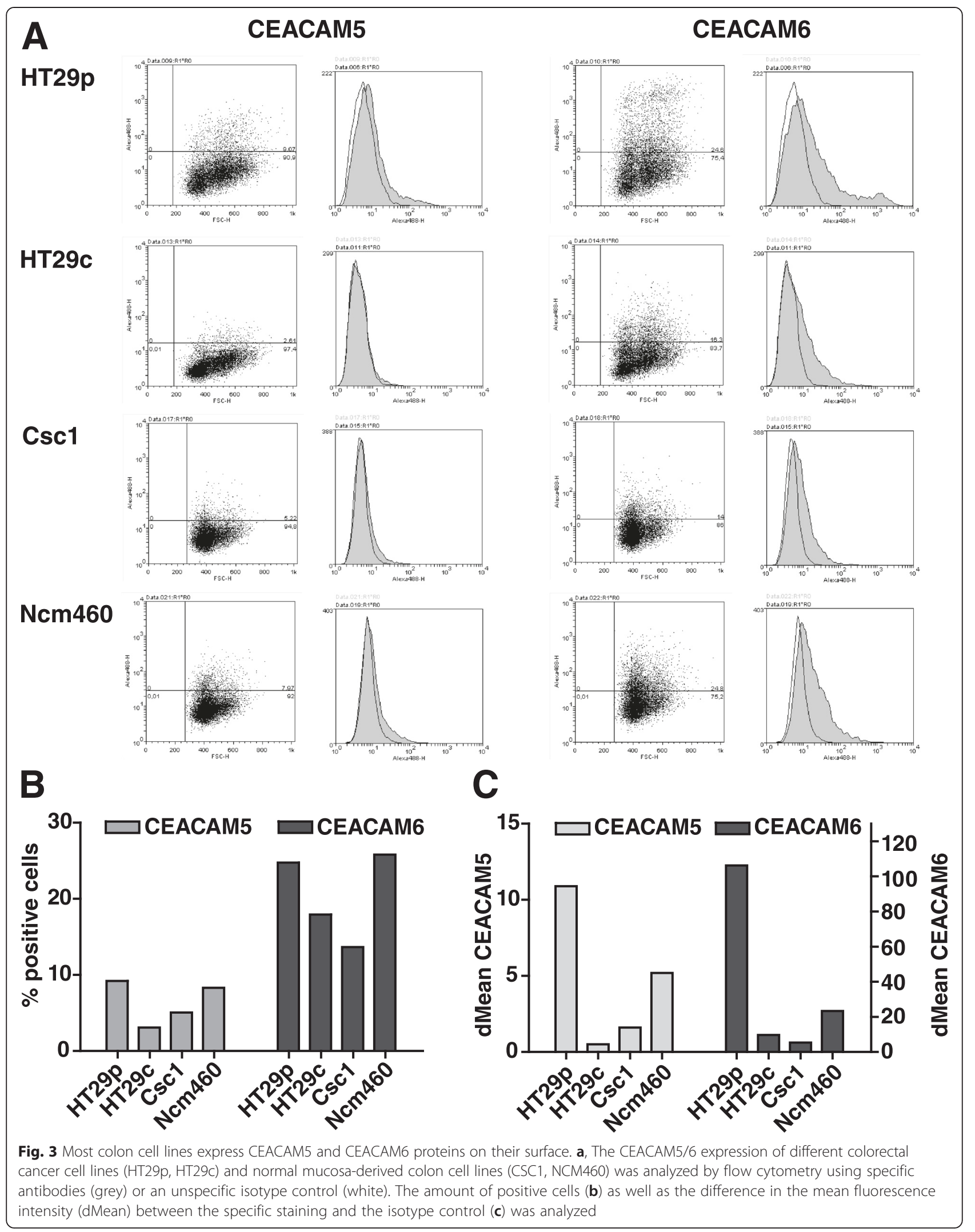


clearly increased its expression of CEACAM5 and CEACAM6 with higher confluency (Fig. 2). SW480 cells did not express any CEACAM5/6 (data not shown).

On the protein level, we analyzed the CEACAM5/6 surface expression by flow cytometry (Fig. 3). In a given cell population, only a fraction of cells was positive for CEACAM5 and CEACAM6. Similar to our findings on the mRNA level, HT29p cells had the highest surface protein expression level (represented by the difference in the mean fluorescence intensity between isotype controlstained and CEACAM5/6-stained cells), as well as the highest percentage of positive cells (Fig. 3). NCM460 cells showed a similar amount of positive cells, but the expression level was lower than in HT29p cells. Again, HT29c cells

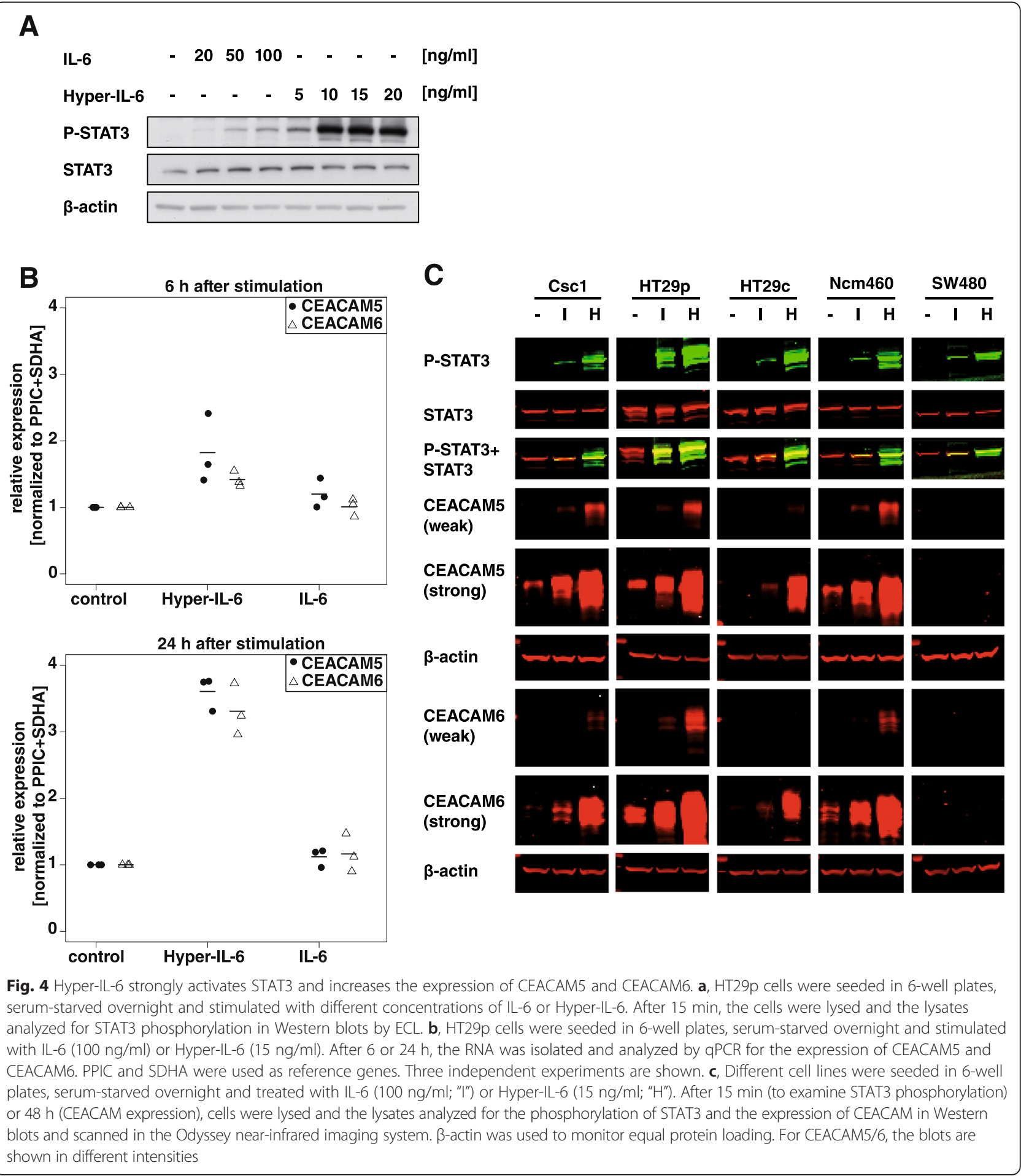


exhibited a lower expression of CEACAM5/6 than HT29p, and SW480 did not show any surface expression.

\section{IL-6 trans-signaling upregulates the expression of CEACAM5 and CEACAM6 in colon cancer cells}

To study the effect of IL- 6 classic and trans-signaling, cells were stimulated either with IL-6 or Hyper-IL-6 (consisting of human IL-6 linked by a flexible peptide chain to the soluble form of the IL-6 receptor) at different concentrations. For this preliminary experiment, the cell line HT29p was chosen, because it did not show an endogenous IL-6 expression (Fig. 1a). As one of the earliest steps in the IL- 6 signaling cascade, we analyzed the phosphorylation of STAT3. While IL-6 only weakly activated STAT3, Hyper-IL-6 led to a strong phosphorylation even at low concentrations (Fig. 4a). This suggests that HT29p cells express IL-6R only in small amounts and are not very responsive to IL-6.

To answer the question whether IL-6 signaling leads to an upregulation of the CEACAM5/6 expression, we treated HT29p cells with IL-6 (100 ng/ml) or Hyper-IL-6 (15 ng/ml) and analyzed the CEACAM5/6 expression on the mRNA level after 6 and $24 \mathrm{~h}$. After $6 \mathrm{~h}$, only slight changes were observed, but after $24 \mathrm{~h}$, expression of CEACAM5 and CEACAM6 was clearly increased by HyperIL-6 stimulation (Fig. 4b). In contrast, IL-6 only led to slight changes in the CEACAM5/6 expression (Fig. 4b).

We confirmed this finding at the protein level in the two normal mucosa-derived cell lines (CSC1, NCM460) and two representative colorectal cancer cell lines which express CEACAM5 and CEACAM6 (HT29p, HT29c) (Fig. 4c). STAT3 was strongly phosphorylated by HyperIL-6 in all of these cell lines. Stimulation with IL-6 led to a much weaker STAT3 phosphorylation, although its concentration was much higher than that of Hyper-IL-6. Consequently, the CEACAM expression was not as strongly increased as with Hyper-IL-6. The cell line
SW480 did not express any detectable CEACAM5 or CEACAM6. This also did not change after treatment with IL-6 or Hyper-IL-6, indicating that CEACAM expression is not inducible by IL-6 de novo, but is typically stimulated by IL-6 trans-signaling (Fig. 4c). While the relationship between IL-6 and CEACAM5 expression was not clear in the literature, our data suggest that IL-6 leads to a small increase in the expression of CEACAM5 and CEACAM6, but that this increase is much stronger when IL-6 trans-signaling occurs. This may be due to the low IL-6R expression. Furthermore, we show here for the first time that CEACAM6 is upregulated by IL-6 trans-signaling.

\section{The phosphorylation of STAT3 is necessary for the Hyper-IL-6-mediated increase in CEACAM5/6}

To analyze whether the Hyper-IL-6-mediated increase in CEACAM5/6 expression depends on the phosphorylation of STAT3, HT29p cells were pre-treated with FLLL31, a small molecule STAT3 inhibitor derived from curcumin [37]. Subsequently, the cells were stimulated either with normal serum-free medium or with HyperIL-6. FLLL31 clearly inhibited the Hyper-IL-6-induced phosphorylation of STAT3 (Fig. 5a) and the increase in CEACAM5/6 expression (Fig. 5b).

IL-6 trans-signaling stabilizes hypoxia-inducible factor 1a (HIF-1a), and chemical stabilization of HIF-1a upregulates CEACAM5/6

IL-6 was shown to increase the expression of HIF- $1 \alpha$ at the protein synthesis level [38]. HIF-1 $\alpha$, in turn, was described to upregulate CEACAM5 and CEACAM6 [39, 40]. Therefore, we tested whether the observed (Hyper-)IL-6-mediated CEACAM5/6 increase could be due to an increased HIF-1 $\alpha$ level.

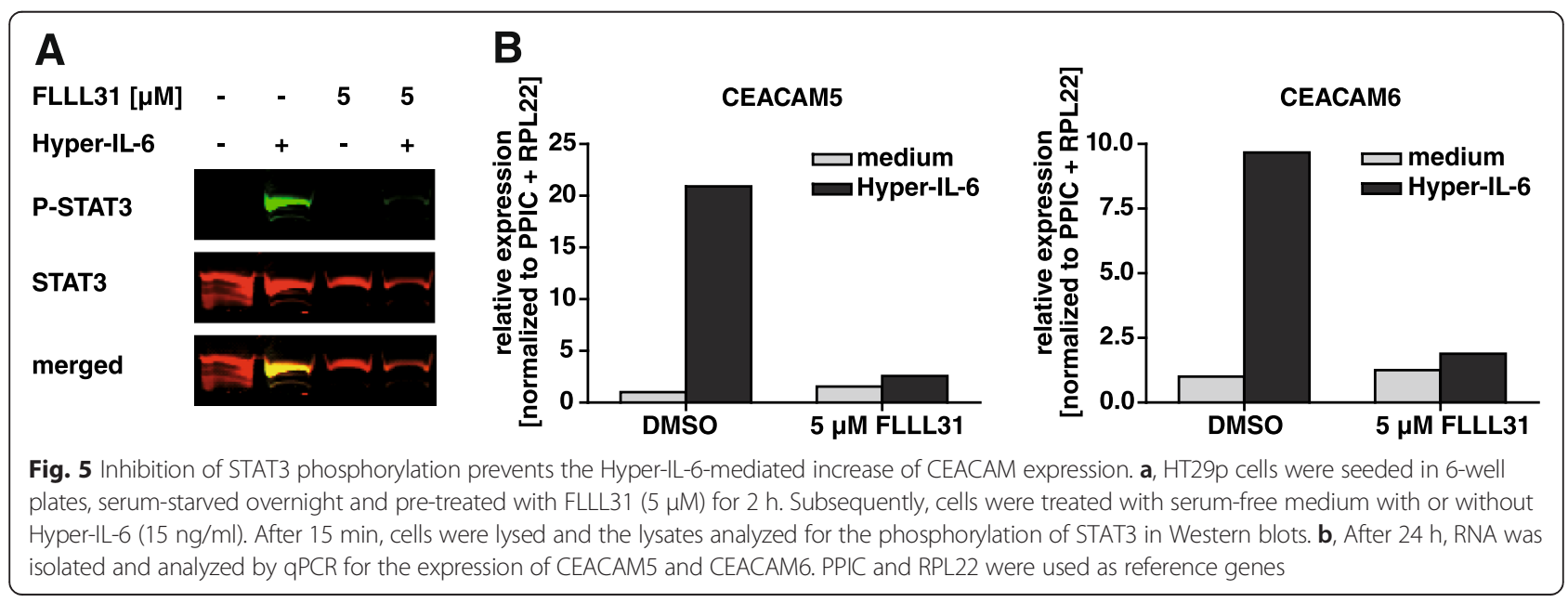




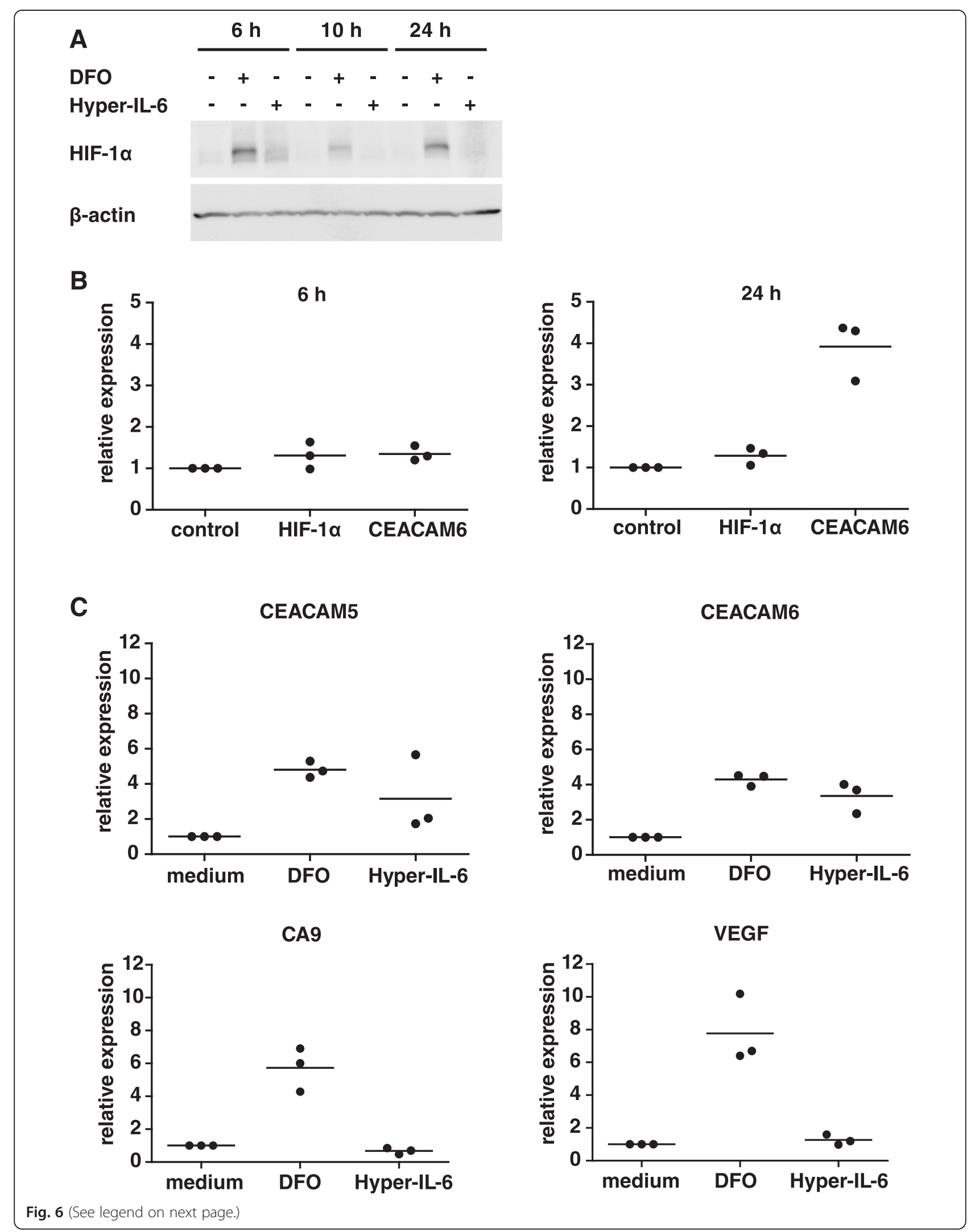


(See figure on previous page.)

Fig. 6 Hyper-IL-6 increases the expression of HIF-1a on the protein level. a, HT29p cells were treated with Hyper-IL-6 (15 ng/ml) or with 100 HM of deferoxamine mesylate (DFO) as a positive control. Cells were lysed at the indicated time points and analyzed by Western blotting for the expression of HIF-1a. b. HT29p cells were treated with Hyper-IL-6 (15 ng/ml). After 6 or 24 h, RNA was extracted and analyzed by qPCR for the expression of HIF-1a. The expression of CEACAM6 was used as a positive control. Three independent experiments are shown. $\mathbf{c}$, HT29p cells were seeded in 6-well plates. Cells were serum-starved and subsequently stimulated with DFO (100 $\mu \mathrm{M})$ or with Hyper-IL-6 (15 ng/ml). After 24 h, RNA was extracted and analyzed by qPCR for the expression of CA9, VEGF, CEACAM5 and CEACAM6. Three independent experiments are shown

We confirmed that treatment of HT29p cells with Hyper-IL- 6 indeed increased HIF-1 $\alpha$ on the protein level after $6 \mathrm{~h}$ of incubation. At later time points, this difference decreased (Fig. 6a). On the mRNA level, we did not detect a significant upregulation of HIF- $1 \alpha$ mRNA (Fig. 6b). HIF-1 $\alpha$ is chemically stabilized by the iron chelator deferoxamine mesylate (DFO). DFO inhibits prolyl hydroxylases, which degrade HIF-1 $\alpha$ [41]. We used DFO as a control to confirm that HIF-1 $\alpha$ leads to an upregulation of CEACAMs. Treatment of HT29p cells with DFO led to a clear upregulation of CEACAM5 as well as of CEACAM6 (Fig. 6c). The classic HIF-1 $\alpha$ target genes CA9 and VEGF were used as positive controls. However, they were only upregulated by DFO but not by Hyper-IL-6, although Hyper-IL-6 increased the HIF-1 $\alpha$ protein level in these cells. In summary, these data support the notion that HIF- $1 \alpha$ plays a role in the Hyper-IL-6-induced CEACAM5/6 upregulation, but further studies are necessary to understand the complex mechanism of regulation.

\section{Discussion}

In this study, we show that IL-6 trans-signaling significantly upregulates the expression of CEACAM5 and CEACAM6 in CRC cells. IL-6 trans-signaling is known to be important for the development of CRC [6]. We show that some CRC cells constitutively express IL-6, whereas all of the tested cell lines express IL-6R and gp130 on the mRNA level. However, cells only weakly responded to stimulation with IL-6. In comparison, IL-6 trans-signaling (induced by Hyper-IL-6 mimicking the IL-6/sIL-6R complex) strongly phosphorylated STAT3 and led to a significant increase in CEACAM5 and CEACAM6 expression. Interestingly, cell lines originally derived from normal mucosa [28] also expressed IL-6, IL-6R and gp130. This is consistent with other studies, demonstrating expression of IL-6 and mIL-6R in intestinal epithelial cells [42].

IL-6 classic signaling weakly phosphorylated STAT3 and increased the expression of CEACAM5 and CEACAM6 in different colon cell lines. In comparison, IL-6 transsignaling had a much stronger effect. This may be due to a low IL-6R expression on the cell surface. The differential IL-6R expression could also be the explanation why some previous studies described an effect of IL-6 on the CEACAM expression [21, 22] while others did not [23]. Accordingly, we also observed a STAT3 phosphorylation and a CEACAM5/6 upregulation by classic signaling in the pancreatic cell line Colo357, which obviously expressed sufficient amounts of IL-6R (Additional file 1: Figure S1).

In HT29p cells, the observed influence of IL-6 transsignaling on the CEACAM expression depended on the phosphorylation of STAT3, as an inhibitor of the STAT3 phosphorylation blocked the Hyper-IL-6-mediated CEACAM increase. Moreover, Hyper-IL-6 led to an increase in HIF-1 $\alpha$ levels. Interestingly, this increase was only observed on the protein level but not on mRNA level, an effect previously described for IL-6. Briggs showed in his doctoral thesis that IL- 6 increases the rate of HIF- $1 \alpha$ synthesis (translation) rather than the rate of transcription [43], and STAT3 was shown to inhibit the degradation of HIF-1 $\alpha$ [44]. Increased translation seems to be a common mechanism for HIF- $1 \alpha$ increase after stimulation with growth/oncogenic stimuli [44-46].

Stabilization of HIF- $1 \alpha$ by the hypoxia mimetic deferoxamine mesylate (DFO) led to an upregulation of CEACAM5 and CEACAM6. This suggested that HIF-1 $\alpha$ might be involved in the Hyper-IL-6-mediated CEACAM5/6 upregulation. Moreover, STAT3 and HIF- $1 \alpha$ had previously been shown to interact in transcriptional complexes to regulate the expression of HIF-1 $\alpha$ target genes [47-50]. However, the classical HIF-1 $\alpha$ target genes CA9 and VEGF were not upregulated by Hyper-IL-6 in our settings. Therefore, further studies are necessary to elucidate the detailed mechanism of transcriptional regulation of CEACAM5 and CEACAM6.

\section{Conclusions}

In summary, we show in this study that IL-6 transsignaling increases the expression of CEACAM5 and CEACAM6 in colon cells. This may be important for tumorigenesis, as CEACAM5 and CEACAM6 are involved in adhesion, migration, invasion and metastasis [17]. This study provides further support for inhibiting IL-6 trans-signaling as a clinical therapeutic strategy for colorectal cancer [51-53]. 


\section{Additional file}

Additional file 1: Figure S1. IL-6 influences the expression of CEACAM5 and CEACAM6 in the pancreatic cancer cell line Colo357. Colo357 cells were treated with IL-6 $(100 \mathrm{ng} / \mathrm{ml})$ in serum-free medium or with the anti-IL-6Rantibody tocilizumab (10 or $100 \mu \mathrm{g} / \mathrm{ml}$ ) in serum-containing medium to block endogenous IL-6 signaling for $24 \mathrm{~h}$. RNA was isolated and qPCR performed to analyze the expression of CEACAM5/6. (PDF $95 \mathrm{~kb}$ )

\section{Abbreviations}

BSA: bovine serum albumin; CEA: carcinoembryonic antigen; CEACAM: carcinoembryonic antigen-related cell adhesion molecule; CAC: colitis-associated cancer; CRC: colorectal cancer; DFO: deferoxamine mesylate; DMSO: dimethyl sulfoxide; ECL: electrochemiluminescence; ELISA: enzyme-linked immunosorbent assay; FBS: fetal bovine serum; gp130: glycoprotein 130; HIF-1a: hypoxia-inducible factor 1a; HRP: horseradish peroxidase; IBD: inflammatory bowel disease; IL6: interleukin-6; IL-6R: IL-6 receptor; MAPK: mitogen-activated protein kinase; mIL-6R: membrane-bound form of the IL-6R; NK cells: natural killer cells; PBS: phosphate-buffered saline; PI3K: phosphatidylinositol-4,5-bisphosphate 3kinase; PMA: phorbol-12-myristate-13-acetate; PPIC: peptidylprolyl isomerase C; qPCR: quantitative PCR; RPL22: ribosomal protein L22; RT-PCR: reverse transcriptase polymerase chain reaction; RIPA: radioimmunoprecipitation assay; RT: room temperature; SDHA: succinate dehydrogenase complex subunit A flavoprotein; sIL-6R: soluble IL-6 receptor; STAT3: signal transducer and activator of transcription-3; TBS: tris-buffered saline.

\section{Competing interests}

SRJ is an inventor on gp130 patents owned by CONARIS and a shareholder of the CONARIS Research Institute AG (Kiel, Germany), which is commercially developing sgp130Fc. GHW is employed by the CONARIS Research Institute AG and an inventor on gp130 patents owned by CONARIS. All other authors declare no competing financial interests.

\section{Authors' contributions}

$\mathrm{RH}$ performed all experiments, analyzed the data and wrote the manuscript ST, HK, SRJ and RH designed the study. HK, SRJ and GW contributed substantially to the experimental design and the interpretation of data. All authors critically revised the manuscript. All authors read and approved the final manuscript.

\section{Acknowledgment}

This work was supported by the Deutsche Forschungsgemeinschaft (DFG; Cluster of Excellence "Inflammation at Interfaces", RH, HK + SRJ; SFB841, project C1; SFB877, project A1, SRJ). The authors would like to thank Eva Simon for providing primer sequences and Christian Roeder for fruitful discussions.

\section{Author details}

${ }^{1}$ Division of Molecular Oncology, Institute for Experimental Cancer Research, University Hospital Schleswig-Holstein, 24105 Kiel, Germany. ${ }^{2}$ CONARIS Research Institute AG, Kiel, Germany. ${ }^{3}$ Section Biomedical Imaging, Department of Radiology and Neuroradiology, University Hospital Schleswig-Holstein, Kiel, Germany. ${ }^{4}$ Institute of Biochemistry, Christian-Albrechts-University, Kiel, Germany.

Received: 17 July 2015 Accepted: 23 November 2015 Published online: 16 December 2015

\section{References}

1. Malvezzi M, Bertuccio P, Levi F, La Vecchia C, Negri E. European cancer mortality predictions for the year 2014. Ann Oncol. 2014;25:1650-6.

2. Raskov H. Colorectal carcinogenesis-update and perspectives. WJG. 2014;20:18151

3. American Cancer Society. Colorectal Cancer facts \& Figures 2014-2016. Atlanta, Georgia; 2014. http://www.cancer.org/research/cancerfactsstatistics/ colorectal-cancer-facts-figures. (Accessed Nov 24, 2015).

4. Lutgens MWMD, van Oijen MGH, van der Heijden GJMG, Vleggaar FP, Siersema PD, Oldenburg B. Declining risk of colorectal cancer in inflammatory bowel disease: an updated meta-analysis of population-based cohort studies. Inflamm Bowel Dis. 2013;19:789-99.

5. Yashiro M. Ulcerative colitis-associated colorectal cancer. WJG. 2014;20:16389.

6. Waldner MJ, Neurath MF. Master regulator of intestinal disease: IL-6 in chronic inflammation and cancer development. Semin Immunol. 2014;26:75-9.

7. Scheller J, Chalaris A, Schmidt-Arras D, Rose-John S. The pro- and anti-inflammatory properties of the cytokine interleukin-6. Biochim Biophys Acta. 1813;2011:878-88.

8. Rincon M. Interleukin-6: from an inflammatory marker to a target for inflammatory diseases. Trends Immunol. 2012;33:571-7.

9. Scheller J, Garbers C, Rose-John S. Interleukin-6: from basic biology to selective blockade of pro-inflammatory activities. Semin Immunol. 2014;26:2-12.

10. Chalaris A, Schmidt-Arras D, Yamamoto K, Rose-John S. Interleukin-6 trans-signaling and colonic cancer associated with inflammatory bowel disease. Dig Dis. 2012;30:492-9.

11. Belluco C, Nitti D, Frantz M, Toppan P, Basso D, Plebani M, et al. Interleukin-6 blood level is associated with circulating carcinoembryonic antigen and prognosis in patients with colorectal cancer. Ann Surg Oncol. 2000;7:133-8.

12. Nakagoe T, Tsuji T, Sawai T, Tanaka K, Hidaka S, Shibasaki S, et al. The relationship between circulating interleukin- 6 and carcinoembryonic antigen in patients with colorectal cancer. Anticancer Res 2003;23:3561-64

13. Gold P, Freedman SO. Specific carcinoembryonic antigens of the human digestive system. J. Exp. Med. 1965;122:467-81.

14. VukobratBijedic Z, HusicSelimovic A, Sofic A, Bijedic N, Bjelogrlic I, Gogov B, et al. Cancer Antigens (CEA and CA 19-9) as Markers of Advanced Stage of Colorectal Carcinoma. Med Arh. 2013;67:397.

15. Polat E, Duman U, Duman M, Atici A, Reyhan E, Dalgic T, et al. Diagnostic value of preoperative serum carcinoembryonic antigen and carbohydrate antigen 19-9 in colorectal cancer. Curr Oncol. 2014;21:1.

16. Zheng C, Feng J, Lu D, Wang P, Xing S, Coll J, et al. A Novel Anti-CEACAM5 Monoclonal Antibody, CC4, Suppresses Colorectal Tumor Growth and Enhances NK Cells-Mediated Tumor Immunity. PLoS One. 2011;6:e21146.

17. Beauchemin N, Arabzadeh A. Carcinoembryonic antigen-related cell adhesion molecules (CEACAMs) in cancer progression and metastasis. Cancer Metastasis Rev. 2013;32:643-71.

18. Schölzel S, Zimmermann W, Schwarzkopf G, Grunert F, Rogaczewski B, Thompson J. Carcinoembryonic antigen family members CEACAM6 and CEACAM7 are differentially expressed in normal tissues and oppositely deregulated in hyperplastic colorectal polyps and early adenomas. Am J Pathol. 2000;156:595-605.

19. Jantscheff P, Terracciano L, Lowy A, Glatz-Krieger K, Grunert F, Micheel B, et al. Expression of CEACAM6 in resectable colorectal cancer: a factor of independent prognostic significance. J Clin Oncol. 2003;21:3638-46.

20. Ilantzis C, DeMarte L, Screaton RA, Stanners CP. Deregulated expression of the human tumor marker CEA and CEA family member CEACAM6 disrupts tissue architecture and blocks colonocyte differentiation. Neoplasia. 2002:4:151-63

21. Ullmann CD, Schlom J, Greiner JW. Interleukin-6 increases carcinoembryonic antigen and histocompatibility leukocyte antigen expression on the surface of human colorectal carcinoma cells. J Immunother. 1992;12:231-41.

22. Dansky-Ullmann C, Salgaller M, Adams S, Schlom J, Greiner JW. Synergistic effects of IL-6 and IFN-gamma on carcinoembryonic antigen (CEA) and HLA expression by human colorectal carcinoma cells: role for endogenous IFN-beta. Cytokine. 1995;7:118-29.

23. Verhaar MJ, Damen CA, Zonnenberg BA, Blijham GH. In vitro upregulation of carcinoembryonic antigen expression by combinations of cytokines. Cancer Lett. 1999;139:67-73.

24. Vogel I, Shen $Y$, Soeth E, Juhl H, Kremer B, Kalthoff $H$, et al. A human carcinoma model in athymic rats reflecting solid and disseminated colorectal metastases. Langenbecks Arch Surg. 1998;383:466-73.

25. Wang M, Vogel I, Kalthoff H. Correlation between metastatic potential and variants from colorectal tumor cell line HT-29. World J Gastroenterol. 2003;9:2627-31.

26. Morgan RT, Woods LK, Moore GE, Quinn LA, McGavran L, Gordon SG. Human cell line (COLO 357) of metastatic pancreatic adenocarcinoma. Int J Cancer. 1980;25:591-8.

27. Mann B, Gelos M, Siedow A, Hanski ML, Gratchev A, llyas M, et al. Target genes of beta-catenin-T cell-factor/lymphoid-enhancer-factor signaling in human colorectal carcinomas. Proc Natl Acad Sci U S A. 1999;96:1603-8. 
28. Moyer MP, Manzano LA, Merriman RL, Stauffer JS, Tanzer LR. NCM460, a normal human colon mucosal epithelial cell line. In Vitro Cell Dev Biol Anim. 1996:32:315-7

29. Gearing DP, Ziegler SF, Comeau MR, Friend D, Thoma B, Cosman D, et al. Proliferative responses and binding properties of hematopoietic cells transfected with low-affinity receptors for leukemia inhibitory factor, oncostatin M, and ciliary neurotrophic factor. Proc Natl Acad Sci U S A. 1994:91:1119-23.

30. Vollmer P, Oppmann B, Voltz N, Fischer M, Rose-John S. A role for the immunoglobulin-like domain of the human IL-6 receptor. Intracellular protein transport and shedding. Eur J Biochem. 1999;263:438-46.

31. van Dam M, Müllberg J, Schooltink H, Stoyan T, Brakenhoff JP, Graeve L, et al. Structure-function analysis of interleukin- 6 utilizing human/murine chimeric molecules. Involvement of two separate domains in receptor binding. J Biol Chem. 1993;268:15285-90.

32. Fischer M, Goldschmitt J, Peschel C, Brakenhoff JP, Kallen KJ, Wollmer A, et al. I. A bioactive designer cytokine for human hematopoietic progenitor cell expansion. Nat Biotechnol. 1997;15:142-5.

33. Schmiegel WH, Kalthoff H, Arndt R, Gieseking J, Greten H, Klöppel G, et al. Monoclonal antibody-defined human pancreatic cancer-associated antigens. Cancer Res. 1985:45:1402-7.

34. Nap M, Hammarström ML, Börmer $\mathrm{O}$, Hammarström S, Wagener C, Handt S, et al. Specificity and affinity of monoclonal antibodies against carcinoembryonic antigen. Cancer Res. 1992;52:2329-39.

35. Stauffer JS, Manzano LA, Balch GC, Merriman RL, Tanzer LR, Moyer MP. Development and characterization of normal colonic epithelial cell lines derived from normal mucosa of patients with colon cancer. Am J Surg. 1995:169:190-5. discussion 195-6.

36. Garbers C, Jänner N, Chalaris A, Moss ML, Floss DM, Meyer D, et al. Species specificity of ADAM10 and ADAM17 proteins in interleukin-6 (IL-6) trans-signaling and novel role of ADAM10 in inducible IL-6 receptor shedding. J Biol Chem. 2011;286:14804-11.

37. Lin L, Hutzen B, Zuo M, Ball S, Deangelis S, Foust E, et al. Novel STAT3 phosphorylation inhibitors exhibit potent growth-suppressive activity in pancreatic and breast cancer cells. Cancer Res. 2010;70:2445-54.

38. Xu Q, Briggs J, Park S, Niu G, Kortylewski M, Zhang S, et al. Targeting Stat3 blocks both HIF-1 and VEGF expression induced by multiple oncogenic growth signaling pathways. Oncogene. 2005;24:5552-60.

39. Kokkonen N, Ulibarri IF, Kauppila A, Luosujärvi H, Rivinoja A, Pospiech H, et al. Hypoxia upregulates carcinoembryonic antigen expression in cancer cells. Int J Cancer. 2007;121:2443-50.

40. Denizot J, Desrichard A, Agus A, Uhrhammer N, Dreux N, Vouret-Craviari V, et al. Diet-induced hypoxia responsive element demethylation increases CEACAM6 expression, favouring Crohn's disease-associated Escherichia coli colonisation. Gut. 2015;64:428-37.

41. Siddiq A, Ayoub IA, Chavez JC, Aminova L, Shah S, LaManna JC, et al. Hypoxia-inducible Factor Prolyl 4-Hydroxylase Inhibition: A TARGET FOR NEUROPROTECTION IN THE CENTRAL NERVOUS SYSTEM. J Biol Chem. 2005:280:41732-43.

42. Waetzig GH, Rose-John S. Hitting a complex target: an update on interleukin-6 trans-signalling. Expert Opin Ther Targets. 2012;16:225-36.

43. Briggs J. Role of stat3 in regulating hif-1alpha expression and tumor angiogenesis. Graduate Theses and Dissertations. 2005. http:// scholarcommons.usf.edu/etd/2792. (Accessed July 9, 2015).

44. Jung JE, Kim HS, Lee CS, Shin Y, Kim Y, Kang G, et al. STAT3 inhibits the degradation of HIF-1a by pVHL-mediated ubiquitination. Exp Mol Med. 2008:40:479

45. Laughner E, Taghavi P, Chiles K, Mahon PC, Semenza GL. HER2 (neu) Signaling Increases the Rate of Hypoxia-Inducible Factor 1 (HIF-1) Synthesis: Novel Mechanism for HIF-1-Mediated Vascular Endothelial Growth Factor Expression. Mol Cell Biol. 2001;21:3995-4004.

46. Semenza GL. Targeting HIF-1 for cancer therapy. Nat Rev Cancer. 2003;3:721-32.

47. Gray MJ, Zhang J, Ellis LM, Semenza GL, Evans DB, Watowich SS, et al. HIF-1a, STAT3, CBP/p300 and Ref-1/APE are components of a transcriptional complex that regulates Src-dependent hypoxia-induced expression of VEGF in pancreatic and prostate carcinomas. Oncogene. 2005;24:3110-20.

48. Jung JE, Lee $H G$, Cho IH, Chung DH, Yoon S, Yang YM, et al. STAT3 is a potential modulator of HIF-1-mediated VEGF expression in human renal carcinoma cells. FASEB J. 2005;19:1296-8.
49. Oh M, Park H, Kim N, Park S, Park I, Kim I. Hypoxia-inducible Factor-1 Enhances Haptoglobin Gene Expression by Improving Binding of STAT3 to the Promoter. J Biol Chem. 2011;286:8857-65.

50. Pawlus MR, Wang L, Hu C. STAT3 and HIF1a cooperatively activate HIF1 target genes in MDA-MB-231 and RCC4 cells. Oncogene. 2013;33:1670-9.

51. Becker C, Fantini MC, Schramm C, Lehr HA, Wirtz S, Nikolaev A, et al. TGF-beta suppresses tumor progression in colon cancer by inhibition of IL-6 trans-signaling. Immunity. 2004;21:491-501.

52. Grivennikov S, Karin E, Terzic J, Mucida D, Yu G, Vallabhapurapu S, et al. IL-6 and Stat3 are required for survival of intestinal epithelial cells and development of colitis-associated cancer. Cancer Cell. 2009;15:103-13.

53. Matsumoto S, Hara T, Mitsuyama K, Yamamoto M, Tsuruta O, Sata M, et al. Essential roles of IL-6 trans-signaling in colonic epithelial cells, induced by the IL-6/soluble-IL-6 receptor derived from lamina propria macrophages, on the development of colitis-associated premalignant cancer in a murine model. J Immunol. 2010;184:1543-51.

54. Nakanishi H, Yoshioka K, Joyama S, Araki N, Myoui A, Ishiguro S, et al. Interleukin-6/soluble interleukin-6 receptor signaling attenuates proliferation and invasion, and induces morphological changes of a newly established pleomorphic malignant fibrous histiocytoma cell line. Am J Pathol. 2004:165:471-80.

55. Sherwin JRA, Smith SK, Wilson A, Sharkey AM. Soluble gp130 is upregulated in the implantation window and shows altered secretion in patients with primary unexplained infertility. J Clin Endocrinol Metab. 2002;87:3953-60

56. de Jonge HJM, Fehrmann RSN, de Bont ESJM, Hofstra RMW, Gerbens F, Kamps WA, et al. Evidence based selection of housekeeping genes. PLoS One. 2007;2, e898.

57. Roda G, Dahan S, Mezzanotte L, Caponi A, Roth-Walter F, Pinn D, et al. Defect in CEACAM family member expression in Crohn's disease IECS is regulated by the transcription factor SOX9. Inflamm Bowel Dis. 2009;15:1775-83

\section{Submit your next manuscript to BioMed Central and we will help you at every step:}

- We accept pre-submission inquiries

- Our selector tool helps you to find the most relevant journal

- We provide round the clock customer support

- Convenient online submission

- Thorough peer review

- Inclusion in PubMed and all major indexing services

- Maximum visibility for your research

Submit your manuscript at www.biomedcentral.com/submit 